\title{
Positive Youth Development in Aboriginal Physical Activity and Sport: A Systematic Review
}

\author{
Mark W. Bruner ${ }^{1} \cdot$ Sean Hillier ${ }^{2}$ Colin P. T. Baillie ${ }^{3} \cdot$ Lynn F. Lavallee $^{4}$. \\ Brenda G. Bruner ${ }^{1} \cdot$ Katharine Hare $^{5} \cdot$ Robert Lovelace $^{6} \cdot$ Lucie Lévesque $^{3}$
}

Received: 19 October 2015/Accepted: 7 December 2015/Published online: 19 December 2015

(C) Springer International Publishing 2015

\begin{abstract}
Physical activity and, more specifically, sport, can positively impact the body, mind, heart, and spirit of Aboriginal youth. Despite enthusiasm for these benefits, there is a paucity of published information reviewing the relationship between sport and physical activity, and Aboriginal youth development. The purpose of this systematic review was to provide an overview of research examining Aboriginal youth development in physical activity and sport settings. A review of literature was conducted up until July 2015 using 10 key databases and non-indexed Indigenous journals. Articles meeting the following criteria were retained: (a) involved an Indigenous population; (b) explored, either through empirical research or conceptualization, the notion of "positive youth development" and "physical activity", "sport", or "recreation". Thirty-five articles out of the 10,039 documents identified met the inclusion criteria. Thematic analysis revealed that physical activity and sport provides many positive developmental opportunities for Aboriginal youth
\end{abstract}

Mark W. Bruner

markb@nipissingu.ca

1 School of Physical and Health Education, Nipissing University, 100 College Drive, Box 5002, North Bay, ON P1B 8L7, Canada

2 Policy Studies, Ryerson University, Toronto, Canada

3 School of Kinesiology and Health Studies, Queen's University, Kingston, Canada

4 School of Social Work, Ryerson University, Toronto, Canada

5 Motivate Canada, Ottawa, Canada

6 Department of Global Development Studies, Queen's University, Kingston, Canada to thrive (e.g., to incorporate Indigenous values and traditional ways, to strengthen resiliency, to improve all aspects of health, and to promote culture and education), as well as possible negative developmental experiences (e.g., racism, bullying, and exclusion). To our knowledge, this article presents the first review of research examining Aboriginal youth development in sport and physical activity and highlights how the activity context can shape Aboriginal youth development.

Keywords Indigenous - Aboriginal - Adolescence . Physical activity · Youth development $\cdot$ Health

\section{Introduction}

Participation in physical activity through purposeful movement of the body is associated with a number of physical and psychosocial benefits (Côté and Fraser-Thomas 2011; Eccles et al. 2003; Eime et al. 2013; Holt 2008). Over the past decade, there has been an increased focus on understanding the psychosocial development of youth through sport and physical activity engagement (Holt and Neely 2011; Taylor et al. 2015). Much of this research has drawn from developmental systems theory (Lerner and Castellino 2002) and developmental psychology, and has undertaken a positive youth development perspective (PYD). PYD is conceptualized as a strength-based rather than deficit-based perspective of adolescent development that recognizes that all young people hold the potential for positive, successful, and healthy development (Lerner et al. 2005). This concept has been embraced by adults who work with youth (teachers, coaches) and there is considerable empirical evidence to support the salient role of 
sport and physical activity in fostering PYD (Eccles et al. 2003; Fredricks and Eccles 2006, 2008).

To date, there is a lack of published literature reporting on PYD programs specific to Aboriginal youth populations (Kana'iaupuni 2005; Mayeda et al. 2001). We know little about the relationship between sport and physical activity and Aboriginal (defined as persons in Canada who identify as First Nations (FN), Inuit or Métis; Dussault et al. 1996) youth development, or about how sport and physical activity might be leveraged to address the historical adversity faced by Aboriginal youth in Canada. This represents a significant gap in knowledge because the legacy of several forms of colonization by European settlers in Canada has impacted the well-being and development of Aboriginal youth in Canada (Gracey and King 2009). Aboriginal youth development has been mortgaged by multigenerational gaps in the transmission of Indigenous culture, language and identity (McShane et al. 2009; Truth and Reconciliation Commission of Canada 2015). A comprehensive understanding of PYD among Aboriginal youth must include historical and cultural perspectives. An emerging body of literature has highlighted the benefits of physical activity, especially with organized sport and recreational opportunities, for Aboriginal youth. For example, sport has been used to teach personal and social values such as honesty, courage and respect and been reported to positively impact the body, mind, heart and spirit of Aboriginal youth (Cargo et al. 2007; Lavallée and Lévesque 2013; Lockard 2000). Further, sport has been advocated as a valuable medium for holistic development in realms extending beyond sport (Blodgett et al. 2008). Through participation in sport, Aboriginal athletes have reported greater preparation and support in their pursuit of ambitions (Blodgett et al. 2008). More broadly, physical activity has also been identified as having the potential to reduce self-harm, levels of substance abuse and improve social cohesion in Indigenous communities (Cunningham and Beneforti 2005).

Despite these benefits, Aboriginal youth have also reported some negative experiences associated with physical activity and sport participation. For example, a study by Blodgett et al. (2008) investigating the sport experience of elite Aboriginal athletes revealed systematic racism faced by young Aboriginal athletes. Other studies have indicated that the participation of Aboriginal youth in sport has often led to animalistic portrayals of these athletes (Bloom 1996). The identified negative experiences alongside others noted among Euro-American populations (e.g., stress, anxiety, burnout; Wiersma 2000) highlight the importance of considering both positive and negative outcomes associated with Aboriginal youth sport and physical activity participation.

\section{Current Study}

The mixed evidence on Aboriginal youth development through physical activity, and more specifically, sport, highlights a need to conduct a thorough review of the literature to guide the development and implementation of Aboriginal physical activity and sport programs and interventions to enhance PYD. The current study will thus address the following research questions: How are physical activity and sport related to Aboriginal youth development? How can Aboriginal youth development be conceptualized through physical activity and sport? Thus, the purpose was to provide a systematic review of research examining Aboriginal youth development in physical activity and sport settings.

\section{Method}

The selection of documents for the review on youth development in Aboriginal physical activity and sport was conducted in three phases: Phase 1-Indexed peer-reviewed literature, Phase 2-Non-indexed Indigenous literature, and Phase 3-Grey literature. In Phases 1 and 2, document inclusion criteria were: (a) involved an Indigenous population; (b) explored, either through empirical research or conceptualization, the notion of "positive youth development"; (c) specific to the physical activity or sport domain; (d) peer-reviewed article; and (e) English or French language. Inclusion criteria in Phase 3 were the same as Phases 1 and 2 with the exception of criteria d. Given the paucity of published research on PYD in Aboriginal youth, we included all types of research studies (i.e., intervention and observational) in each of the three phases.

\section{Phase 1: Indexed Peer-Reviewed Literature}

Phase 1 began with a search for peer-reviewed articles focusing on the following populations: "Aboriginal", OR "First Nations", OR "Métis", OR "Inuit", OR "American Indian", OR "Indian" (omitting Indian from India), OR "Native American", OR "Indigenous" OR "Indians of North America" OR "Pacific Islander" OR "Alaskan Native" AND contexts "Sport" or "Recreation" or "Physical Activity", while using the following combinations of topic terms, "Positive youth development" OR "Leadership" OR "Athlete development" OR "Prosocial" OR "Life skills" (development) OR "Sport outcomes" OR "Psychosocial development" OR "resiliency" OR "empowerment" in the article title, identified keywords, or abstract. Given the breadth of the 
topic and the diverse terminology used to describe this population, it was important that this literature search use an array of encompassing population search terms that focused on a variety of Aboriginal identities from around the world [i.e., in a Canadian context ("Aboriginal", "First Nations", "Métis", "Inuit"), an American context ("Native American" "Indian", "Pacific Islander"), and terms used internationally ("Indigenous")]. The initial search was conducted in July-September, 2012 and updated in July 2015 examining all published literature until this date using 10 databases: (a) Academic Search Premier, (b) Education Research Complete, (c) ERIC, (d) MedLine, (e) SportDiscus (f) ProQuest Dissertations and Thesis (g) ProQuest Nursing and Allied Health Source, (h) ProQuest Sociology, (i) PsychINFO, (j) Canadian Research Index (Table 1).

\section{Phase 2: Indigenous Peer-Reviewed Journal Search}

Phase 2 of the review constituted a further pursuit of peer-reviewed articles in Indigenous journals that were not indexed in Phase 1 databases. Many of the academic indices do not include Indigenous specific journals. As such, a list of non-indexed Indigenous peer-reviewed journals was compiled (Table 2). Twenty-two additional peer-reviewed journals were identified from this source. Three more journals were identified by research team members, yielding a total of 25 journals in this phase. The 25 journals were then examined using the same search terms and inclusion criteria outlined in Phase 1 (Table 1). The initial search was conducted in OctoberDecember 2012 and updated in July, 2015. Material published in the journals since inception were included in the search.

\section{Phase 3: Grey Literature Search}

Phase 3 of the review consisted of grey literature searches and personal correspondence with experts in Aboriginal youth development. Phase 3 began in December of 2012 and was updated in July 2015 with a search for non-peer reviewed journal articles, periodicals, government documents, and websites using the search terms in Phase 1 and 2 through Google (Table 1). The Google search was limited to the first four pages of results as per established protocols reflecting typical patterns of viewing Google results (Jansen and Spink 2006; Jetha et al. 2011) Indigenous agency and organization documents, and documents identified by research team members (i.e., who knew of specific organizations and communities doing work in the area of Aboriginal youth development and/or Aboriginal sport) were also collected. As a part of the validation process for Phase 3, two experts in Aboriginal youth development independently reviewed the list of articles and websites identified by the research team. Once articles in all three Phases had been retrieved using the population and topic terms outlined above, abstracts of articles were read to determine if some inclusion criteria were met. Articles containing abstracts that met at least some of the inclusion criteria were then read in their entirety and subjected to a full content review; articles containing all of the inclusion criteria were retained for analysis.

\section{Article Analysis}

The articles retained in all three Phases were classified by methodology (i.e., quantitative, qualitative, or mixed method), and independently coded according to themes identified within them that positively and negatively affect

Table 1 Systematic review search strategy

\begin{tabular}{|c|c|c|c|c|}
\hline Phase & Content & Database(s) & Key terms & Inclusion criteria \\
\hline 1 & $\begin{array}{l}\text { Indexed } \\
\text { Peer- } \\
\text { Reviewed } \\
\text { Journals }\end{array}$ & $\begin{array}{l}\text { 1. Academic Search Premier; } 2 . \\
\text { Education Research Complete; } 3 . \\
\text { ERIC; 4. MedLine; 5. SportDiscus; } \\
\text { 6. ProQuest Dissertations and } \\
\text { Thesis; 7. ProQuest Nursing \& } \\
\text { Allied Health Source; 8. ProQuest } \\
\text { Sociology; 9. PsychINFO; } 10 . \\
\text { Canadian Research Index }\end{array}$ & \multirow{3}{*}{ 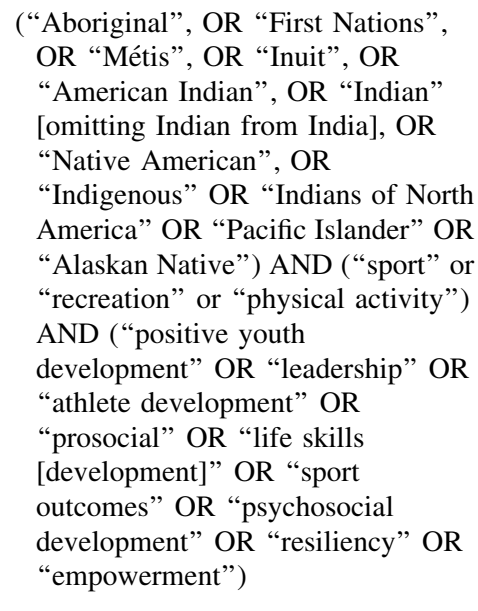 } & \multirow[t]{2}{*}{$\begin{array}{l}\text { (a) Involved an Indigenous } \\
\text { population; (b) Explored, either } \\
\text { through empirical research or } \\
\text { conceptualization, the notion of } \\
\text { 'positive youth development'; } \\
\text { (c) Specific to the sport or physica } \\
\text { activity domain; (d) peer-reviewed } \\
\text { article; (e) English or French } \\
\text { language }\end{array}$} \\
\hline 2 & $\begin{array}{l}\text { Non-Indexed } \\
\text { Peer- } \\
\text { Reviewed } \\
\text { Indigenous } \\
\text { Journals }\end{array}$ & Table 2 & & \\
\hline 3 & $\begin{array}{l}\text { Non Peer- } \\
\text { Reviewed } \\
\text { Grey } \\
\text { Literature }\end{array}$ & Google search & & Same as above, excluding (d) \\
\hline
\end{tabular}


Table 2 Non-indexed indigenous journals searched during phase 2

\begin{tabular}{ll}
\hline Journal Name & Inaugural publication year \\
\hline AlterNative: An International Journal of Indigenous Scholarship & 2005 \\
Australian Indigenous Law Review & 1996 \\
American Indian Culture and Research Journal & 1974 \\
BC Studies: The British Columbian Quarterly & 1969 \\
Cultural Survival Quarterly & 1972 \\
Decolonization: Indigeneity, Education and Society & 2012 \\
First Peoples Child \& Family Review & 2004 \\
International Journal of Circumpolar Health & 1972 \\
Journal of Aboriginal Health & 2004 \\
Journal of Ethnopharmacology & 1979 \\
Indigenous Policy Journal & 1989 \\
Indigenous Law Journal & 2002 \\
International Journal of Critical Indigenous Studies & 2008 \\
International Indigenous Policy Journal & 2010 \\
Journal of Indigenous Research & 2011 \\
MAI Review & 2006 \\
Native Social Work Journal & 1997 \\
Native South & 2008 \\
Pimatisiwin: A Journal of Aboriginal and Indigenous Community Health & 2003 \\
Plains Anthropologist & 1955 \\
Settler Colonial Studies & 2011 \\
Tribal Law Journal & 1998 \\
Te Kaharoa & 2008 \\
UCLA's Indigenous Peoples Journal of Law, Culture \& Resistance & 2004 \\
Wicazo Sa Review & 1985 \\
\hline
\end{tabular}

youth development in an Indigenous population by two coders using NVivo 10 software. Following the recommendations of Braun and Clarke (2006), a six-phase method of identifying, analyzing and reporting themes within the data was used: (1) familiarizing oneself with the articles, (2) generating initial codes, (3) searching for youth development themes in the articles, (4) reviewing themes with the other coder, (5) defining and counting themes, and (6) producing the findings for the manuscript. Any discrepancies in the coding of the articles were resolved through discussion between the two coders and another author. Consensus was obtained for all articles coded.

\section{Results}

Initially, using the keywords and aforementioned study search criteria, 10,039 documents were identified for this systematic review. Figure 1 presents the stages of article identification and selection. Table 3 provides a description of the 35 articles retained for coding. The majority of the articles used a qualitative approach $(n=15)$ (Blodgett

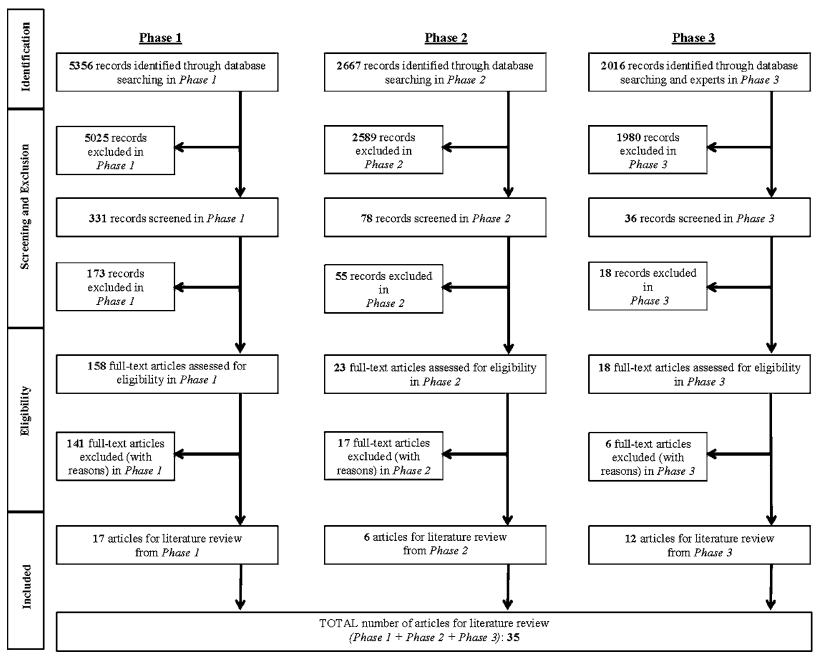

Fig. 1 Stages of article identification and selection

et al. 2008; Bloom 1996; Brown 2015; Brown and Kraft 2008; Cunningham and Beneforti 2005; Donahue 1997; Keith 1999; Marchand 2011; McHugh et al. 2013a, b; 
Table 3 Articles investigating aboriginal youth development through sport and physical activity

\begin{tabular}{|c|c|c|c|c|c|c|c|}
\hline Phase & First author & Year & Title & Journal name & $\begin{array}{l}\text { Methods } \\
\text { classification } \\
\text { (intervention } \\
\text { study) }\end{array}$ & $\begin{array}{l}\text { Youth age } \\
\text { range (when } \\
\text { specified) }\end{array}$ & $\begin{array}{l}\text { Themes } \\
\text { present }\end{array}$ \\
\hline 1 & Blodgett, A. & 2008 & $\begin{array}{l}\text { From practice to praxis community- } \\
\text { based strategies for aboriginal } \\
\text { youth sport }\end{array}$ & $\begin{array}{l}\text { Journal of Sport } \\
\text { \& Social } \\
\text { Issues }\end{array}$ & Qualitative & N/A & $\begin{array}{c}\text { T, E, N, } \\
\text { B }\end{array}$ \\
\hline 1 & Brown, L. & 2008 & $\begin{array}{l}\text { Active Living as an institutional } \\
\text { challenge: lessons from the Robert } \\
\text { Wood Johnson Foundation's } \\
\text { "Celebrate Fitness" Program }\end{array}$ & $\begin{array}{l}\text { Journal of } \\
\text { Health } \\
\text { Politics, } \\
\text { Policy, and } \\
\text { Law }\end{array}$ & $\begin{array}{l}\text { Qualitative } \\
\text { (Intervention) }\end{array}$ & N/A & $\begin{array}{l}\mathrm{HF}, \mathrm{E}, \\
\mathrm{B}\end{array}$ \\
\hline 1 & Cunningham, J. & 2005 & $\begin{array}{l}\text { Investigating indicators for } \\
\text { measuring the health and social } \\
\text { impact of sport and recreation } \\
\text { Programs in Australian indigenous } \\
\text { communities }\end{array}$ & $\begin{array}{l}\text { International } \\
\text { Review for the } \\
\text { Sociology of } \\
\text { Sport }\end{array}$ & Qualitative & N/A & $\begin{array}{l}\mathrm{R}, \mathrm{HF} \\
\mathrm{ED}\end{array}$ \\
\hline 1 & Donovan, D. & 2015 & $\begin{array}{l}\text { Healing of the Canoe: preliminary } \\
\text { results of a culturally grounded } \\
\text { intervention to prevent substance } \\
\text { abuse and promote tribal identity } \\
\text { for native youth in Two Pacific } \\
\text { Northwest Tribe }\end{array}$ & $\begin{array}{l}\text { American } \\
\text { Indian and } \\
\text { Alaskan } \\
\text { Native Mental } \\
\text { Health } \\
\text { Research }\end{array}$ & $\begin{array}{l}\text { Quantitative } \\
\text { (Intervention) }\end{array}$ & Grades 9-12 & $\mathrm{T}, \mathrm{R}, \mathrm{E}$ \\
\hline 1 & Feinstein, S. & 2009 & $\begin{array}{l}\text { Resiliency and Native American } \\
\text { Teenagers }\end{array}$ & $\begin{array}{l}\text { Reclaiming } \\
\text { Children and } \\
\text { Youth }\end{array}$ & $\begin{array}{l}\text { Mixed } \\
\text { Methods }\end{array}$ & Grades $9-12$ & $\begin{array}{r}\mathrm{T}, \mathrm{R}, \\
\mathrm{ED}\end{array}$ \\
\hline 1 & Gray, A. & 2003 & $\begin{array}{l}\text { Fitness, dietary intake, and body mass } \\
\text { index in urban Native American } \\
\text { youth }\end{array}$ & $\begin{array}{l}\text { Journal of the } \\
\text { American } \\
\text { Dietetic } \\
\text { Association }\end{array}$ & Quantitative & $5-18$ years & $\mathrm{HF}, \mathrm{B}$ \\
\hline 1 & Keith, S. & 1999 & Native American women in sport & $\begin{array}{l}\text { Journal of } \\
\text { Physical } \\
\text { Education, } \\
\text { Recreation \& } \\
\text { Dance }\end{array}$ & Qualitative & N/A & $\begin{array}{r}\mathrm{T}, \mathrm{HF}, \\
\mathrm{E}, \mathrm{B}\end{array}$ \\
\hline 1 & Kiran, A. & 2010 & $\begin{array}{l}\text { Traditional indigenous games } \\
\text { promoting physical activity and } \\
\text { cultural connectedness in primary } \\
\text { schools-cluster randomised } \\
\text { control trial }\end{array}$ & $\begin{array}{l}\text { Health } \\
\text { Promotion } \\
\text { Journal of } \\
\text { Australia }\end{array}$ & $\begin{array}{l}\text { Quantitative } \\
\text { (Intervention) }\end{array}$ & $9-12$ years & $\mathrm{T}, \mathrm{ED}$ \\
\hline 1 & Marchand, D. & 2011 & $\begin{array}{l}\text { Circle of courage infusion into the } \\
\text { Alberta Indigenous games } 2011\end{array}$ & $\begin{array}{l}\text { Reclaiming } \\
\text { Children and } \\
\text { Youth }\end{array}$ & Qualitative & N/A & $\mathrm{T}, \mathrm{E}$ \\
\hline 1 & McHugh, T. & $\begin{array}{c}2013 a, \\
b\end{array}$ & $\begin{array}{l}\text { An exploration of the meanings of } \\
\text { sport to Urban aboriginal youth }\end{array}$ & $\begin{array}{l}\text { Qualitative } \\
\text { Research in } \\
\text { Sport, } \\
\text { Exercise and } \\
\text { Health }\end{array}$ & Qualitative & $12-15$ years & $\begin{array}{l}\mathrm{T}, \mathrm{HF} \\
\mathrm{E}, \mathrm{N} \\
\mathrm{B}\end{array}$ \\
\hline 1 & Moilanen, K. & 2014 & $\begin{array}{l}\text { Extracurricular activity availability } \\
\text { and participation and substance use } \\
\text { among American Indian } \\
\text { adolescents }\end{array}$ & $\begin{array}{l}\text { Journal of } \\
\text { Youth and } \\
\text { Adolescence }\end{array}$ & Quantitative & 12-19 years & $\mathrm{R}$ \\
\hline 1 & Ritchie, S. & 2010 & $\begin{array}{l}\text { Developing a culturally relevant } \\
\text { outdoor leadership training program } \\
\text { for aboriginal youth }\end{array}$ & $\begin{array}{l}\text { Journal of } \\
\text { Experiential } \\
\text { Education }\end{array}$ & Qualitative & N/A & $\begin{array}{l}\mathrm{T}, \mathrm{R}, \mathrm{E}, \\
\mathrm{B}\end{array}$ \\
\hline 1 & Skinner, K. & 2006 & $\begin{array}{l}\text { Barriers and supports for healthy } \\
\text { eating and physical activity for first } \\
\text { nation youths in Northern Canada }\end{array}$ & $\begin{array}{l}\text { International } \\
\text { Journal of } \\
\text { Circumpolar } \\
\text { Health }\end{array}$ & Qualitative & Grades 6-8 & $\begin{array}{l}\text { HF, ED, } \\
\text { E, B }\end{array}$ \\
\hline
\end{tabular}


Table 3 continued

\begin{tabular}{|c|c|c|c|c|c|c|c|}
\hline Phase & First author & Year & Title & Journal name & $\begin{array}{l}\text { Methods } \\
\text { classification } \\
\text { (intervention } \\
\text { study) }\end{array}$ & $\begin{array}{l}\text { Youth age } \\
\text { range (when } \\
\text { specified) }\end{array}$ & $\begin{array}{l}\text { Themes } \\
\text { present }\end{array}$ \\
\hline 1 & Teufel, N. & 1998 & $\begin{array}{l}\text { Development of a primary prevention } \\
\text { program: insight gained in the Zuni } \\
\text { diabetes prevention program }\end{array}$ & $\begin{array}{l}\text { Clinical } \\
\text { Pediatrics }\end{array}$ & $\begin{array}{l}\text { Quantitative } \\
\text { (Intervention) }\end{array}$ & $13.2-21.2$ years & HF, ED \\
\hline 1 & Tighe, J. & 2012 & $\begin{array}{l}\text { Alive and kicking goals: preliminary } \\
\text { findings from a Kimberly suicide } \\
\text { prevention program }\end{array}$ & $\begin{array}{l}\text { Advances in } \\
\text { Mental Health }\end{array}$ & $\begin{array}{l}\text { Qualitative } \\
\text { (Intervention) }\end{array}$ & N/A & $\begin{array}{l}\mathrm{R}, \mathrm{ED}, \\
\mathrm{E}\end{array}$ \\
\hline 1 & Weaver, H. & 2010 & $\begin{array}{l}\text { Healthy living in two worlds: testing } \\
\text { a wellness curriculum for Urban } \\
\text { Native Youth }\end{array}$ & $\begin{array}{l}\text { Child } \\
\text { Adolescence } \\
\text { and Social } \\
\text { Work Journal }\end{array}$ & $\begin{array}{l}\text { Quantitative } \\
\text { (Intervention) }\end{array}$ & $9-13$ years & $\begin{array}{l}\text { T, HF, } \\
\text { ED }\end{array}$ \\
\hline 1 & Young, N. & 2013 & $\begin{array}{l}\text { A Process for Creating the Aboriginal } \\
\text { Children's Health and Well-Being } \\
\text { Measure (ACHWM) }\end{array}$ & $\begin{array}{l}\text { Canadian } \\
\text { Journal of } \\
\text { Public Health }\end{array}$ & Qualitative & $8.2-17.7$ years & $\mathrm{T}, \mathrm{HF}$ \\
\hline 2 & Bloom, J. & 1996 & $\begin{array}{l}\text { "Show what an Indian can do": } \\
\text { sports, memory, ethnic identity at } \\
\text { federal Indian boarding schools }\end{array}$ & $\begin{array}{l}\text { Journal of } \\
\text { American } \\
\text { Indian } \\
\text { Education }\end{array}$ & Qualitative & N/A & $\mathrm{T}, \mathrm{E}, \mathrm{N}$ \\
\hline 2 & Critchley, K.A. & 2006 & $\begin{array}{l}\text { Personal Health practices around } \\
\text { physical activity as perceived by the } \\
\text { aboriginal children of Prince } \\
\text { Edward Island }\end{array}$ & $\begin{array}{l}\text { Journal of } \\
\text { Aboriginal } \\
\text { Health }\end{array}$ & $\begin{array}{l}\text { Mixed } \\
\text { Methods }\end{array}$ & $6-18$ years & HF, ED \\
\hline 2 & Donahue, $\mathrm{P}$. & 1997 & $\begin{array}{l}\text { New warriors, new legends: } \\
\text { basketball in three Native American } \\
\text { works of fiction }\end{array}$ & $\begin{array}{l}\text { American } \\
\text { Indian } \\
\text { Research and } \\
\text { Culture } \\
\text { Journal }\end{array}$ & Qualitative & N/A & $\mathrm{T}, \mathrm{E}$ \\
\hline 2 & McHugh, T. & $\begin{array}{c}2013 a, \\
b\end{array}$ & $\begin{array}{l}\text { Enhancing the relevance of physical } \\
\text { activity research by engaging } \\
\text { aboriginal peoples in the research } \\
\text { process }\end{array}$ & Pimatisiwin & Qualitative & 14-18 years & $\begin{array}{r}\text { T, HF, } \\
\text { E, B }\end{array}$ \\
\hline 2 & Nicholls, S. & 2007 & $\begin{array}{l}\text { Sport as a tool for HIV/AIDS } \\
\text { education: a potential catalyst for } \\
\text { change }\end{array}$ & Pimatisiwin & Qualitative & N/A & $\begin{array}{l}\mathrm{HF}, \mathrm{ED} \\
\mathrm{E}\end{array}$ \\
\hline 2 & Simmonds, H. & 2014 & Te Kete Whanaketanga - Rangatahi & MAI Review & $\begin{array}{l}\text { Mixed } \\
\text { Methods }\end{array}$ & $12-18$ years & $\begin{array}{l}\text { T, HF, } \\
\text { ED, E }\end{array}$ \\
\hline 3 & Brown, D. & 2015 & Ride for future generations & $\begin{array}{l}\text { Native Peoples } \\
\text { Magazine }\end{array}$ & Qualitative & N/A & $\mathrm{R}$ \\
\hline 3 & $\begin{array}{l}\text { Indigenous } \\
\text { Wellness Group, } \\
\text { Lavallée, L. }\end{array}$ & 2013 & $\begin{array}{l}\text { Six Nations/Ohsweken running club } \\
\text { pilot program final report }\end{array}$ & & $\begin{array}{l}\text { Quantitative } \\
\text { (Intervention) }\end{array}$ & $9-14$ years & $\begin{array}{l}\mathrm{T}, \mathrm{R}, \\
\mathrm{HF}, \mathrm{E}\end{array}$ \\
\hline 3 & $\begin{array}{l}\text { Government of } \\
\text { Saskatchewan. }\end{array}$ & 2008 & $\begin{array}{l}\text { Saskatchewan children and youth } \\
\text { participation in sport, recreation } \\
\text { and cultural activities }\end{array}$ & & $\begin{array}{l}\text { Mixed } \\
\text { Methods }\end{array}$ & 5-19 years & $\begin{array}{r}\text { T, HF, } \\
\text { E, B }\end{array}$ \\
\hline 3 & $\begin{array}{l}\text { Government of } \\
\text { Ontario }\end{array}$ & 2010 & $\begin{array}{l}\text { Helping improve quality of life for } \\
\text { aboriginal youth }\end{array}$ & & $\begin{array}{l}\text { Mixed } \\
\text { Methods } \\
\text { (Intervention) }\end{array}$ & N/A & $\mathrm{HF}, \mathrm{E}$ \\
\hline 3 & Lavallée, L. & 2009 & $\begin{array}{l}\text { Health and social impacts and fitness } \\
\text { testing at the } 2008 \text { North American } \\
\text { Indigenous Games }\end{array}$ & & Quantitative & N/A & $\begin{array}{l}\text { T, R, } \\
\text { HF, E, } \\
\text { ED }\end{array}$ \\
\hline 3 & Navara, G. & 2009 & $\begin{array}{l}\text { CARA Program Evaluation-Nipissing } \\
\text { First Nation }\end{array}$ & & $\begin{array}{l}\text { Mixed } \\
\text { Methods } \\
\text { (Intervention) }\end{array}$ & N/A & $\begin{array}{l}\text { R, HF, } \\
\text { E, B }\end{array}$ \\
\hline
\end{tabular}


Table 3 continued

\begin{tabular}{|c|c|c|c|c|c|c|c|}
\hline Phase & First author & Year & Title & Journal name & $\begin{array}{l}\text { Methods } \\
\text { classification } \\
\text { (intervention } \\
\text { study) }\end{array}$ & $\begin{array}{l}\text { Youth age } \\
\text { range (when } \\
\text { specified) }\end{array}$ & $\begin{array}{l}\text { Themes } \\
\text { present }\end{array}$ \\
\hline 3 & Praxis Consulting & 2014 & $\begin{array}{l}2014 \text { North American indigenous } \\
\text { games evaluation report }\end{array}$ & & $\begin{array}{l}\text { Mixed } \\
\text { Methods }\end{array}$ & N/A & $\begin{array}{l}\mathrm{T}, \mathrm{R}, \\
\mathrm{HF}, \mathrm{E}, \\
\mathrm{ED}\end{array}$ \\
\hline 3 & Right to Play & 2015 & PLAY Program & & $\begin{array}{l}\text { Quantitative } \\
\text { (Intervention) }\end{array}$ & N/A & $\mathrm{E}$ \\
\hline 3 & $\begin{array}{l}\text { World Leisure and } \\
\text { Recreation } \\
\text { Association } \\
\text { Professional } \\
\text { Services (WPS) }\end{array}$ & 1998 & $\begin{array}{l}\text { The } 1998 \text { Arctic Winter Games: a } \\
\text { study of the benefits of participation }\end{array}$ & & $\begin{array}{l}\text { Mixed } \\
\text { Methods }\end{array}$ & N/A & $\begin{array}{l}\text { T, R, } \\
\text { HF, E }\end{array}$ \\
\hline 3 & $\begin{array}{l}\text { World Leisure } \\
\text { Professional } \\
\text { Services (WPS) }\end{array}$ & 2000 & $\begin{array}{l}2000 \text { Arctic Winter Games } \\
\text { Whitehorse: personal and social } \\
\text { benefits of participation }\end{array}$ & & Quantitative & N/A & $\begin{array}{l}\mathrm{T}, \mathrm{R}, \\
\mathrm{HF}, \mathrm{E}\end{array}$ \\
\hline 3 & $\begin{array}{l}\text { World Leisure } \\
\text { Professional } \\
\text { Services (WPS) }\end{array}$ & 2002 & $\begin{array}{l}2002 \text { Arctic Winter Games: Nuuk, } \\
\text { Greenland and Iqaluit, Canada } \\
\text { personal and social benefits of } \\
\text { participation }\end{array}$ & & $\begin{array}{l}\text { Mixed } \\
\text { Methods }\end{array}$ & N/A & $\begin{array}{l}\text { T, R, } \\
\text { HF, E }\end{array}$ \\
\hline 3 & $\begin{array}{l}\text { World Leisure } \\
\text { Professional } \\
\text { Services (WPS) }\end{array}$ & 2010 & $\begin{array}{l}2010 \text { Arctic winter games: personal, } \\
\text { social, and community benefits of } \\
\text { participation }\end{array}$ & & Quantitative & N/A & $\begin{array}{l}\text { T, R, } \\
\text { HF, E }\end{array}$ \\
\hline
\end{tabular}

$T$ tradition, $R$ resiliency, $H F$ overall health and fitness, $E D$ education, $E$ empowerment, $N$ negative outcomes, $B$ barriers

Nicholls and Giles 2007; Ritchie et al. 2010; Skinner et al. 2006; Tighe and McKay 2012; Young et al. 2013) and reported research from Canada $(n=20)$ (Blodgett et al. 2008; Critchley et al. 2006; Government of Ontario 2010; Government of Saskatchewan 2008; Indigenous Wellness Group and Lavallée 2013; Lankford and Neal 1998; Lankford et al. 2010; Lavallée et al. 2009; Marchand 2011; McHugh et al. 2013a, b; Navara et al. 2009; Nicholls and Giles 2007; Praxis Consulting 2014; Right to Play 2015; Ritchie et al. 2010; Skinner et al. 2006; World Leisure Professional Services 2000, 2002; Young et al. 2013). Ten of the reviewed articles described PYD interventions or programs (Brown and Kraft 2008; Donovan et al. 2015; Indigenous Wellness Group and Lavallée 2013; Government of Ontario 2010; Kiran and Knights, 2010; Navara et al. 2009; Right to Play 2015; Teufel and Ritenbaugh 1998; Tighe and McKay 2012; Weaver and Jackson 2010). When specified, the age of youth participants did not exceed 21 years. The most prevalent themes throughout the 35 articles were Empowerment $(n=29)$ (Blodgett et al. 2008; Bloom 1996; Brown and Kraft 2008; Cunningham and Beneforti 2005; Donahue 1997; Donovan et al. 2015; Feinstein et al. 2009; Government of Ontario 2010; Government of Saskatchewan 2008; Indigenous Wellness Group and Lavallée 2013; Keith 1999; Kiran and Knights 2010; Lankford and Neal 1998; Lankford et al. 2010;
Lavallée et al. 2009; Marchand 2011; McHugh et al. 2013a, b; Navara et al. 2009; Nicholls and Giles 2007; Praxis Consulting 2014; Right to Play 2015; Ritchie et al. 2010; Simmonds et al. 2014; Skinner et al. 2006; Teufel and Ritenbaugh 1998; Tighe and McKay 2012; World Leisure Professional Services 2000, 2002), Overall Health and Fitness $(n=23)$ (Brown and Kraft 2008; Critchley et al. 2006; Cunningham and Beneforti 2005; Government of Ontario 2010; Government of Saskatchewan 2008; Gray and Smith 2003; Indigenous Wellness Group and Lavallée 2013; Keith 1999; Lankford and Neal 1998; Lankford et al. 2010; Lavallée et al. 2009; McHugh et al. 2013a, b; Navara et al. 2009; Nicholls and Giles 2007; Praxis Consulting 2014; Simmonds et al. 2014; Skinner et al. 2006; Teufel and Ritenbaugh 1998; Weaver and Jackson 2010; World Leisure Professional Services 2000, 2002; Young et al. 2013), and Traditional Culture and Values $(n=22)$ (Blodgett et al. 2008; Bloom 1996; Donahue 1997; Donovan et al. 2015; Feinstein et al. 2009; Government of Saskatchewan 2008; Indigenous Wellness Group and Lavallée 2013; Keith 1999; Kiran and Knights 2010; Lankford and Neal 1998; Lankford et al. 2010; Lavallée et al. 2009; Marchand 2011; McHugh et al. 2013a, b; Praxis Consulting 2014; Ritchie et al. 2010; Simmonds et al. 2014; Weaver and Jackson 2010; World Leisure Professional Services 2000, 2002; Young et al. 2013). 
Less prevalent themes included Resiliency and Mental Health $(n=16)$ (Brown 2015; Cunningham and Beneforti 2005; Donovan et al. 2015; Feinstein et al. 2009; Indigenous Wellness Group and Lavallée 2013; Lankford and Neal 1998; Lankford et al. 2010; Lavallée et al. 2009; Moilanen et al. 2014; Navara et al. 2009; Praxis Consulting 2014; Ritchie et al. 2010; Simmonds et al. 2014; Tighe and McKay 2012; World Leisure Professional Services 2000, 2002), Education ( $n=12$ ) (Critchley et al. 2006; Cunningham and Beneforti 2005; Feinstein et al. 2009; Kiran and Knights 2010; Lavallée et al. 2009; Nicholls and Giles 2007; Praxis Consulting 2014; Simmonds et al. 2014; Skinner et al. 2006; Teufel and Ritenbaugh 1998; Tighe and McKay 2012; Weaver and Jackson 2010), Barriers ( $n=10$ ) (Blodgett et al. 2008; Brown and Kraft 2008; Government of Saskatchewan 2008; Gray and Smith 2003; Keith 1999; McHugh et al. 2013a, b; Navara et al. 2009; Ritchie et al. 2010; Skinner et al. 2006), and Negative Outcomes $(n=3)$ (Blodgett et al. 2008; Bloom 1996; McHugh et al. 2013a).

\section{Phase 1: Indexed Peer-Reviewed Literature}

As shown in Fig. 1, a search of the 10 databases yielded 17 articles that were retained in Phase 1. One hundred and forty-one articles were excluded as they did not meet the search criteria-either did not focus on youth development (e.g., only mentioned youth development briefly in the discussion section of the article), or did not focus on Indigenous peoples) (see Fig. 1; Table 3).

\section{Phase 2: Indigenous Peer-Reviewed Journal Search}

A search of the non-indexed Indigenous literature yielded six articles that were retained in Phase 2. Seventeen articles were excluded as they either did not focus on youth development (e.g., only mentioned youth development briefly in the discussion section of the article), or did not focus on Indigenous peoples (see Fig. 1; Table 3).

\section{Phase 3: Grey Literature}

Two experts in Aboriginal youth development independently reviewed a total of 88 websites and nine articles identified as relevant by the research team to determine if any others sources had been missed or warranted inclusion. The experts and a member of the research team identified a total of 11 additional organization websites. A search of the suggested websites yielded nine additional articles, bringing the total to 18 articles. After full screening of the 18 identified youth development articles against the study criteria and upon determining if youth development was a major focus of the article, 12 articles were retained in Phase 3. Six articles were excluded as they did not focus on youth development or did not focus on Indigenous peoples (see Fig. 1; Table 3).

\section{Thematic Coding of Included Articles}

Using NVivo 10 software, two coders reviewed the 35 articles retained from all three phases, coding them for themes related to positive youth development in an Indigenous population. Seven themes were identified from the literature: (1) Empowerment, (2) Overall Health and Fitness, (3) Traditional Culture and Values, (4) Education, (5) Barriers to Participation, (6) Resiliency and Mental Health, and (7) Negative Physical Activity and Sport Experiences. Each theme, with associated examples, is described below.

\section{Empowerment}

This theme highlighted Aboriginal youth development leadership skills, overcoming barriers, and building selfworth. For example, Brown and Kraft (2008) documented Celebrate Fitness, a program that provided funding for Native American teens to develop and conduct grassroots initiatives under the supervision of Native American adults. Skinner et al. (2006) also discussed how, in a remote, subarctic First Nations community, the barriers and supports for nutrition and physical activity are heightened and interrelated, thus contributing to much larger social constructs such as empowerment and trust. Skinner et al. (2006) also mentioned that empowerment should be incorporated into the design of public health initiatives as it is a key issue for many Aboriginal communities in Canada. As another example of empowerment, the planning committee for the Alberta Indigenous Games, as discussed by Marchand (2011) recognized the value and potential that practicing Indigenous cultures has on Aboriginal youth. The focal point of the Alberta Indigenous Games was the implementation of the Circle of Courage values, which identified Indigenous traditions that foster social harmony and extraordinary personal achievement. The Circle of Courage values were incorporated into the Games' activities such as sports, music and dance. This philosophy promoted an environment of encouragement, empowerment and acceptance for all. In the Arctic Games reports (Lankford and Neal 1998; Lankford et al. 2010; World Leisure Professional Services 2000, 2002), participants indicated that the experience was fun, provided positive experiences including new friendships and ultimately led to the development of leadership skills. 


\section{Overall Health and Fitness}

This theme highlighted the improvement of Aboriginal youth's physical health (e.g., reduction in diabetes), through becoming active and avoiding health-damaging behaviors such as tobacco use. As an example, the Zuni Diabetes Prevention Program is a community-based primary prevention project designed to reduce the prevalence of diabetes risk factors among high school-aged youth (Teufel and Ritenbaugh 1998). Brown and Kraft (2008) also discussed this theme as they described how empowerment of youth leaders is proving to be an effective approach to improving health and fitness as it reduces tobacco use as well as gun violence. Another example of this theme was presented in Weaver and Jackson's (2010) work, which outlined "Healthy Living in Two Worlds", a project developed to increase physical activity and healthy eating practices while also decreasing or preventing recreational tobacco use in urban, Northeastern Native American youth. In evaluating a running program with First Nations youth, the Indigenous Wellness Group and Lavallée (2013) noted that youth participants improved their physical health along with positive impacts on spiritual, emotional and mental health.

\section{Traditional Culture and Values}

This theme highlighted youth learning about Aboriginal culture and heritage such as language, music, dance, and traditions. As an example, Kiran and Knights (2010) documented the importance of implementing culturally relevant programs in primary schools. The use of a Traditional Indigenous Games program increased retention rates of Indigenous students in schools as well as raised awareness of culture, and improved cultural pride (Kiran and Knights 2010). This theme can also be seen in the work of McHugh et al. (2013a) as the authors discussed the importance of sharing circles in the context of physical activity and the sacred meaning they have for many Indigenous cultures. The work of Young et al. (2013) utilized this theme as their physical activity research was guided by the Medicine Wheel, a culturally relevant model of health and well-being where each quadrant represents a domain of health: spiritual, emotional, physical and mental. Culture and the importance of traditional territories, was also integral for connecting with ancestors and experiencing personal growth as part of the Future Generations Ride horse program (Brown 2015). Lavallée et al. (2009) and Praxis Consulting (2014) conducted research on the 2008 and 2014 North American Indigenous Games respectively and found that sport that incorporates cultural activities and protocols increased cultural pride, awareness and made youth feel more comfortable in the sporting environment.
Resiliency and Mental Health

This theme highlighted suicide prevention, as well as overcoming addictions and depression. As an example, the work of Cunningham and Beneforti 2005) highlighted how sport and recreation has the potential to reduce self-harm and substance use among Aboriginal youth. Further, the article by Feinstein et al. (2009) focused on the importance of opportunities for education and involvement in extracurricular activities as being essential to Native American teenage resiliency. This theme was also present in the work of Ritchie et al. (2010) which recommended mental health promotion strategies developed through a community-driven approach to build Aboriginal youth resilience and cultural identity to prevent suicide. Another example of this theme can be seen through discussion of the project Alive and Kicking Goals! (AKG), a positively-framed suicide prevention peer education project that attempts to improve the provision of mental health services available for at-risk youth in the Kimberly region of Australia (Tighe and McKay 2012).

\section{Education}

This theme highlighted the importance of continuing education for Aboriginal youth, of succeeding in school, and of learning that occurs in programs outside of school. For example, the work of Kiran and Knights (2010) highlighted the protective role an Aboriginal child's sense of connectedness to school plays in preventing problematic behaviors and disengagement with education. Critchley et al. (2006) discussed the importance of physical education programs both during and after school hours as these physical activities may play a significant role in improving Aboriginal students' attitudes, discipline and behaviors.

\section{Barriers to Participation}

This theme highlighted the many real or perceived obstacles that Aboriginal youth struggle with in regard to being physically active. As an example, Skinner et al. (2006) discussed the heightened barriers to healthy living in remote communities where access to physical activity opportunities can be limited. The increased barriers may be due to a lack of capacity and support at the community level. Another example of a barrier to participation in physical activity can be seen in Gray and Smith's (2003) work as they cited neighborhood safety as a major concern for the Aboriginal youth who live in low-income, highcrime areas. In these particular situations, watching television or taking part in other sedentary activities may offer a safe environment as well as relief for the parents (Gray and Smith 2003). The Government of Saskatchewan (2008) 
highlighted the four most frequently cited barriers to being physically active for Aboriginal children as lack of time, cost of participation, lack of local programs and lack of transportation.

\section{Negative Physical Activity and Sport Experience}

This theme encompassed any negative experiences for Aboriginal youth as a result of physical activity participation. Several examples included racism, bullying, and exclusion. In the work of Blodgett et al. (2008), an Aboriginal elite athlete discussed the racism experienced during sport participation; "When people know you're native they're going to say something to you. Those are the times you've got to prepare for. You're not going to fold your tent and go home." Bloom (1996) highlighted the themes of negative sport and physical activity outcomes by drawing on the earlier work of Churchill et al. (1979) who argued that although Indian school athletes were successful, the media images did little for Native Americans themselves other than promote a vision of animalistic, racially defined, purely physical beings.

\section{Discussion}

The period of adolescence has traditionally been framed as one of risk rather than one of opportunity (Takanishi 1993; Jessor 1998; Wolfe et al. 2006). The prevailing paradigm shift toward a PYD perspective now casts youth as resources to be developed rather than problems to be solved (Damon 2004). Childhood and adolescence are considered opportunities for growth in which youth can gain competencies to earn a living, gain knowledge of themselves and their place in society, learn to nurture others, and engage in civic activities (Hamilton et al. 2004; Jones and Lavallee 2009). The goal of optimal youth development is to "enable individuals to live healthy, satisfying, and productive lives as youth and later as adults" (Hamilton et al. 2004, p. 3). This goal is particularly meaningful for Aboriginal youth who have been disadvantaged by the legacy of historic trauma (Aguiar and Halseth 2015). Using this PYD perspective, developmental researchers have reported many positive and some negative outcomes associated with sport and physical activity engagement (Holt and Neely 2011).

The purpose of this review was to provide a systematic review of the research examining Aboriginal youth development in physical activity and sport settings. An analysis of the articles revealed a diversity of themes highlighting how physical activity, especially through sport and recreational opportunities, can shape Aboriginal youth development. With a focus on empowerment, well-being, as well as traditional culture and values, this review illustrates a shift from deficit-reduction approaches that have disempowered youth by labelling behavior as problematic and in need of being "fixed" (Kana'iaupuni 2005; Mayeda et al. 2001). Applied to physical activity and sport, this positive conceptualization may help facilitate the promotion of Indigenous values and the use of local community expertise in program design. For example, the Canoe Journey curriculum developed by Donovan et al. (2015) utilized community members in developing culturallygrounded programming that is preferred, and often more effective, than conventional programs based on mainstream values (Lowe et al. 2011, 2012). As a result, the findings and literature identified provide program developers and practitioners with examples of physical activity and sport that successfully promote Aboriginal youth development in the field and that highlight the importance of culturally meaningful opportunities. For example, the programs outlined in the grey literature illustrate how physical activity and sport programs can be translated to a community context with positive outcomes (Brown 2015; Government of Ontario 2010; Government of Saskatchewan 2008; Lavallée et al. 2009; Navara et al. 2009; Praxis Consulting 2014; Right to Play 2015). The implementation of a multiphased systematic approach encompassing both academic and grey literature provided a deeper understanding of the role of physical activity and sport on Aboriginal youth development.

Despite the novelty of our review, it is important to acknowledge the limitations of the evidence and of the review. Although the search strategy was comprehensive and included grey literature, conference proceedings or articles written in languages other than English or French were not included. In addition, the review did not distinguish between the findings from observation and intervention studies. We recognize that Indigenous communities conduct research and evaluation and that this review of literature does not include grey literature that is not available via the Internet or is unable to be found via our search methods. Finally, we recognize the limitation of language or terminology and how programs that might focus on what we understand to be positive youth development could be conceptualized quite differently in Aboriginal communities. However, the articles included in the review were diverse and used a range of methodological approaches and indicators of youth development through physical activity and sport. Further, the self-reported nature of measures and/or cross-sectional designs used in these studies warrant that the findings be interpreted with caution. 


\section{Conclusion}

To our knowledge, this study represents the first review of the Aboriginal physical activity and sport literature pertaining to positive youth development. This review furthers our understanding of the relationship that physical activity and sport has with Aboriginal youth development. This systematic review also highlights several important recommendations for future research (Grant and Booth 2009). In addition to a need for further qualitative research to deepen the conceptual understanding of Aboriginal youth development through physical activity and sport, there appears to be a need for greater evaluation of Aboriginal physical activity and sport programs. The lack of research in this area may be related to the limited availability of validated measures of Aboriginal youth development through physical activity and sport and an absence of published evidence reporting on the internal and external validity of Aboriginal physical activity sport interventions for enhancing PYD. Future research addressing these areas will enhance our knowledge of how sport and physical activity settings can shape Aboriginal youth development.

Acknowledgments We would like to thank Dr. Claire Crooks and Michele Cook for providing feedback and sharing their knowledge of the Aboriginal youth development literature. The authors also wish to thank Michael Mahkwa Auski, Kevin Emiry, Christine Head, Donna Ivimey, Aaron Paibomsai, and Tristan Tremblay for their assistance with the article collection. This research is supported by a grant from the Canadian Institutes for Health Research (CIHR), Institute of Aboriginal Peoples' Health (IAPH), and Health Canada-First Nations and Inuit Health Branch (FNIHB) (CIHR Grant \#AHI120532)

Author Contributions MWB conceptualized and designed the review, supervised overall data acquisition, conducted the analysis, provided interpretation of findings, drafted the manuscript, has given final approval of the submitted manuscript and agrees to be accountable for all aspects of the work. SH supervised Phase I data acquisition and applied inclusion criteria to screen Phase I documents, critically revised the manuscript and agrees to be accountable for all aspects of the work. CPTB completed Phase II data acquisition and Phase I, II, III data acquisition update and applied inclusion criteria to screen documents, participated in the thematic analysis, helped with interpretation of findings, participated in drafting parts of the manuscript, critically revised the manuscript, has given final approval of the submitted manuscript and agrees to be accountable for all aspects of the work. LFL conceptualized and designed the review, supervised overall data acquisition, critically revised the manuscript, has given final approval of the submitted manuscript and agrees to be accountable for all aspects of the work. BGB conceptualized and designed the review, supervised data acquisition, critically revised the manuscript, has given final approval of the submitted manuscript and agrees to be accountable for all aspects of the work. KH helped conceptualize and design the review, critically revised the manuscript, has given final approval of the submitted manuscript and agrees to be accountable for all aspects of the work. RL helped conceptualize and design the review, critically revised the manuscript, has given final approval of the submitted manuscript and agrees to be accountable for all aspects of the work. LL conceptualized and designed the review, supervised Phase II data acquisition, contributed to data analysis and interpretation of findings, participated in drafting parts of the manuscript, critically revised the manuscript, has given final approval of the submitted manuscript and agrees to be accountable for all aspects of the work.

\section{Compliance with Ethical Standards}

Conflicts of interest The authors report no conflict of interests.

\section{References}

Aguiar, W., \& Halseth, R. (2015). Aboriginal peoples and Historic Trauma: The process of intergenerational transmission. Prince George, BC: National Collaborating Centre for Aboriginal Health.

Blodgett, A. T., Schinke, R. J., Fisher, L. A., Wassengeso George, C., Peltier, D., Ritchie, S., \& Pickard, P. (2008). From practice to praxis: Community-based strategies for Aboriginal youth sport. Journal of Sport and Social Issues, 32(4), 393-414. doi:10.1177/ 0193723508323701.

Bloom, J. (1996). "Show what an Indian can do": Sports, memory, ethnic identity at federal Indian boarding schools. Journal of American Indian Education, 35, 6.

Braun, V., \& Clarke, V. (2006). Using thematic analysis in psychology. Qualitative Research in Psychology, 3(2), 77-101. doi:10.1191/1478088706qp063oa.

Brown, D. (2015). Ride for future generations. Native Peoples Magazine, 28(2), 5.

Brown, L. D., \& Kraft, M. K. (2008). Active living as an institutional challenge: Lessons from the Robert Wood Johnson Foundation's "Celebrate Fitness" program. Journal of Health Politics, Policy and Law, 33(3), 497-523. doi:10.1215/03616878-2008-006.

Cargo, M., Peterson, L., Lévesque, L., \& Macaulay, A. (2007). Perceived wholistic health and physical activity in Kanien'kehá: Ka youth. Pimatisiwin: A Journal of Aboriginal and Indigenous Community Health, 5(1), 87-109.

Churchill, W., Hill, N., \& Barlow, M. (1979). An historical overview of twentieth century Native American athletics. The Indian Historian, 12(4), 22-32.

Côté, J., \& Fraser-Thomas, J. (2011). Youth involvement and positive development in sport. In P. Crocker (Ed.), Sport and exercise psychology: A Canadian perspective (2nd ed., pp. 226-255). Toronto: Pearson Canada Inc.

Critchley, K., Walton, F., Timmons, V., Bryanton, J., McCarthy, M., \& Taylor, J. (2006). Personal health practices around physical activity as perceived by aboriginal children of Prince Edward Island. Journal of Aboriginal Health, 3, 26-33.

Cunningham, J., \& Beneforti, M. (2005). Investigating indicators for measuring the health and social impact of sport and recreation programs in Australian indigenous communities. International Review for the Sociology of Sport, 40, 89-98.

Damon, W. (2004). What is positive youth development? The ANNALS of the American Academy of Political and Social Science, 591(1), 13-24. doi:10.1177/0002716203260092.

Donahue, P. (1997). New warriors, new legends: Basketball in three Native American works of fiction. American Indian Culture and Research Journal, 21(2), 43-60.

Donovan, D., Thomas, L., Sigo, R., Price, L., Lonczak, H., Lawrence, N., et al. (2015). Healing of the canoe: Preliminary results of a culturally grounded intervention to prevent substance abuse and promote tribal identity for Native youth in two Pacific Northwest Tribes. American Indian and Alaska Native Mental Health Research, 22(1), 36. 
Dussault, R., Erasmus, G., \& Royal Commission on Aboriginal Peoples. (1996). Report of the royal commission on aboriginal peoples. Ottawa: Canada Communication Group-Publishing.

Eccles, J. S., Barber, B. L., Stone, M., \& Hunt, J. (2003). Extracurricular activities and adolescent development. Journal of Social Issues, 59(4), 865-889. doi:10.1046/j.0022-4537.2003. 00095.x.

Eime, R. M., Young, J. A., Harvey, J. T., Charity, M. J., \& Payne, W. R. (2013). A systematic review of the psychological and social benefits of participation in sport for adults: Informing development of a conceptual model of health through sport. International Journal of Behavioral Nutrition and Physical Activity, 10, 135. doi:10.1186/1479-5868-10-135.

Feinstein, S., Driving-Hawk, C., \& Baartman, J. (2009). Resiliency and native American teenagers. Reclaiming Children and Youth, $18,12-17$.

Fredricks, J. A., \& Eccles, J. S. (2006). Is extracurricular participation associated with beneficial outcomes? Concurrent and longitudinal relations. Developmental Psychology, 42(4), 698-713. doi:10.1037/0012-1649.42.4.698.

Fredricks, J. A., \& Eccles, J. S. (2008). Participation in extracurricular activities in the middle school years: Are there developmental benefits for African American and European American youth? Journal of Youth and Adolescence, 37, 1029-1043. doi:10.1007/ s10964-008-9309-4.

Government of Ontario, Ministry of Aboriginal Affairs. (2010). Helping improve quality of life for aboriginal youth. Retreived from http://news.ontario.ca/maa/en/2010/11/helping-improvequality-of-life-for-aboriginal-youth.html.

Government of Saskatchewan, Ministry of Tourism, Parks, Culture and Sport. (2008). Saskatchewan children and youth participation in sport, recreation and cultural activities: A re-analysis of data from the 2005 aboriginal participation in sport, culture and recreation study. Regina, Saskatchewan: Ministry of Tourism, Parks, Culture and Sport.

Gracey, M., \& King, M. (2009). Indigenous health part 1: Determinants and disease patterns. The Lancet, 374(9683), 65-75. doi:10.1016/S0140-6736(09)60914-4.

Grant, M. J., \& Booth, A. (2009). A typology of reviews: An analysis of 14 review types and associated methodologies. Health Information \& Libraries Journal, 26(2), 91-108. doi:10.1111/j. 1471-1842.2009.00848.x.

Gray, A., \& Smith, C. (2003). Fitness, dietary intake, and body mass index in urban Native American youth. Journal of the American Dietetic Association, 103(9), 1187-1191. doi:10.1053/jada.2003. 50572.

Hamilton, S., Hamilton, M., \& Pittman, K. (2004). Principles for youth development. In S. Hamilton \& M. Hamilton (Eds.), The youth development handbook: Coming of age in American communities (pp. 3-22). Thousand Oaks, CA: Sage.

Holt, N. (2008). Positive youth development through sport. Oxon: Routledge.

Holt, N., \& Neely, K. (2011). Positive youth development through sport: A review. Revista Iberoamericana de Psicología del Ejerció y el Deporte, 6(2), 299-316.

Indigenous Wellness Group, \& Lavallée, L. (2013). Six Nations/ Ohsweken running club pilot program final report (pp. 1-50). Kahnawake: Québec.

Jansen, B. J., \& Spink, A. (2006). How are we searching the World Wide Web? A comparison of nine search engine transaction logs. Information Processing and Management, 42(1), 248-263. doi:10.1016/j.ipm.2004.10.007.

Jessor, R. (1998). New perspectives on adolescent risk behaviour. New York: Cambridge University Press.

Jetha, A., Faulkner, G., Gorczynski, P., Arbour-Nicitopoulos, K., \& Martin Ginis, K. A. (2011). Physical activity and individuals with spinal cord injury: Accuracy and quality of information on the Internet. Disability and Health Journal, 4(2), 112-120. doi:10.1016/j.dhjo.2010.07.001.

Jones, M., \& Lavallee, D. (2009). Exploring the life skills needs of British adolescent athletes. Psychology of Sport and Exercise, 10(1), 159-167.

Kana'iaupuni, S. M. (2005). Ka'akālai Kū Kanaka: A call for strengths-based approaches from a Native Hawaiian perspective. Educational Researcher, 34(5), 7.

Keith, S. (1999). Native American women in sport. Journal of Physical Education, Recreation and Dance, 70, 47-49.

Kiran, A., \& Knights, J. (2010). Traditional Indigenous Games promoting physical activity and cultural connectedness in primary schools-cluster randomised control trial. Health Promotion Journal of Australia, 21(2), 149-151.

Lankford, S. V., \& Neal, L. L. (1998). The 1998 Arctic Winter Games: A study of the benefits of participation (pp. 1-64). Yellowknife: Northwest Territories.

Lankford, S. V., Neal, L. L., Bonwell, C., Cunningham, A., \& Walter, S. (2010). 2010 Arctic Winter Games: Personal, social, and community benefits of participation (pp. 1-73). Yellowknife: Northwest Territories.

Lavallée, L., \& Lévesque, L. (2013). Two-eyed seeing: physical activity, sport, and recreation promotion in Indigenous communities. In J. Forsyth \& A. Giles (Eds.), Aboriginal peoples and sport in Canada (pp. 206-233). Victoria, BC: UBC Press.

Lavallée, L., Thorne, D., Day, K., Thorne, T., \& Matchiwita, S. (2009). Health and social impacts and fitness testing at the 2008 North American indigenous games (pp. 1-28). Cowichan, BC: North American Indigenous Games Council.

Lerner, R. M., \& Castellino, D. R. (2002). Contemporary developmental theory and adolescence: Developmental systems and applied developmental science. Journal of Adolescent Health, 31(6, Supplement), 122-135. doi:http://dx.doi.org/10.1016/ S1054-139X(02)00495-0

Lerner, R. M., Lerner, J. V., Almerigi, J. B., Theokas, C., Phelps, E., Gestsdottir, S., \& von Eye, A. (2005). Positive youth development, participation in community youth development programs, and community contributions of fifth-grade adolescents: Findings from the first wave of the 4-H study of positive youth development. The Journal of Early Adolescence, 25(1), 17-71. doi: $10.1177 / 0272431604272461$.

Lockard, V. (2000). Native American Sports Council gets honor. From Canku Ota: A newsletter celebrating Native America. Retrieved July 9, 2010, from http://www.turtletrack.org/ Issues00/Co02122000/CO_02122000_sportscouncil.htm

Lowe, J., Liang, H., Riggs, C., \& Henson, J. (2012). Community partnership to affect substance abuse among Native American adolescents. The American Journal of Drug and Alcohol Abuse, $38(5), 6$.

Lowe, J., Riggs, C., \& Henson, J. (2011). Principles for establishing trust when developing a substance abuse intervention with a Native American community. Creative Nursing, 17(2), 6.

Marchand, D. (2011). Circle of courage infusion into the Alberta Indigenous Games 2011. Reclaiming Children and Youth, 20, $59-61$.

Mayeda, D., Chesney-Lind, M., \& Koo, J. (2001). Talking story with Hawai'i's youth: Confronting violent and sexualized perceptions of ethnicity and gender. Youth \& Society, 33(1), 30.

McHugh, T. L. F., Coppola, A. M., \& Sinclair, S. (2013a). An exploration of the meanings of sport to urban Aboriginal youth: A photovoice approach. Qualitative Research in Sport, Exercise and Health, 5(3), 291-311. doi:10.1080/2159676X.2013. 819375.

McHugh, T. L. F., Kingsley, B., \& Coppola, A. (2013b). Enhancing the relevance of physical activity research by engaging 
Aboriginal peoples in the research process. Pimatisiwin: A Journal of Aboriginal and Indigenous Community Health, 11, 293-305.

McShane, K., Smylie, J., \& Adomako, P. (2009). Health of First Nations, Inuit, and Métis Children in Canada. In J. Smylie \& P. Adomako (Eds.), Indigenous children's health report health assessment in action (pp. 11-65). Toronto, ON: Saint Michael's Hospital.

Moilanen, K., Markstrom, C., \& Jones, E. (2014). Extracurricular activity availability and participation and substance use among American Indian adolescents. Journal of Adolescence \& Youth, $43,17$.

Navara, G., Ackerman, M., \& Smith-Chant, B. (2009). CARA Program Evaluation-Nipissing First Nation (Prepared by Community Perspectives on Healthy Development, Trans.) (pp. 109).

Nicholls, S., \& Giles, A. (2007). Sport as a tool for HIV/AIDS education: A potential catalyst for change. Pimatisiwin: A Journal of Aboriginal and Indigenous Community Health, 5, $51-85$.

Praxis Consulting. (2014). 2014 North American indigenous games evaluation report (pp. 1-103). Regina: SK.

Right to Play. (2015). PLAY Program. Retrieved July 16, 2015, from http://www.righttoplay.ca/Learn/ourstory/Pages/PLAY-Program. aspx

Ritchie, S. D., Wabano, M. J., Young, N., Schinke, R., Peltier, D., Battochio, R., \& Russell, K. (2010). Developing a culturally relevant outdoor leadership training program for Aboriginal youth. Journal of Experiential Education, 32(3), 300-304. doi:10.1177/105382590903200315.

Simmonds, H., Harré, N., \& Crengle, S. (2014). Te Kete Whanaketanga - Rangatahi. MAI Journal, 3(3), 16.

Skinner, K., Hanning, R. M., \& Tsuji, L. J. (2006). Barriers and supports for healthy eating and physical activity for First Nation youths in northern Canada. International Journal of Circumpolar Health, 65(2), 148-161.

Takanishi, R. (1993). The opportunities of adolescence-Research, interventions, and policy: Introduction to the special issue. American Psychologist, 48(2), 85-87. doi:10.1037/0003-066X. 48.2.85.
Taylor, P., Davies L., Wells, P., Gilbertson, J., \& Tayleur, W. (2015). A review of the social impacts of culture and sport. Retrieved from https://www.gov.uk/government/uploads/system/uploads/ attachment_data/file/416279/A_review_of_the_Social_Impacts_ of_Culture_and_Sport.pdf

Teufel, N. I., \& Ritenbaugh, C. K. (1998). Development of a primary prevention program: Insight gained in the Zuni Diabetes Prevention Program. Clinical Pediatrics, Philadelphia, 37(2), 131-141.

Tighe, J., \& McKay, K. (2012). Alive and Kicking Goals!: Preliminary findings from a Kimberley suicide prevention program. Advances in Mental Health, 10(3), 240-245. doi:10. 5172/jamh.2012.10.3.240.

Truth and Reconciliation Commission of Canada. (2015). Honouring the truth, reconciling for the future: Summary of the final report of the TRC of Canada (pp. 418). Winnipeg, Manitoba: Truth and Reconciliation Commission of Canada.

Weaver, H. N., \& Jackson, K. F. (2010). Healthy Living in Two Worlds: Testing a wellness curriculum for urban Native youth. Child and Adolescent Social Work Journal, 27(3), 231-244. doi:10.1007/s10560-010-0197-6.

Wiersma, L. D. (2000). Risks and benefits of youth sport specialization: Perspectives and recommendations. Paediatric Exercise Science, 12, 13-22.

Wolfe, D., Jaffe, P., \& Crooks, C. (2006). Adolescent risk behaviours: Why teens experiment and strategies to keep them safe. New Haven, CT: Yale University Press.

World Leisure Professional Services. (2000). 2000 Arctic Winter Games Whitehorse: Personal and social benefits of participation (p. 62). Yellowknife: Northwest Territories.

World Leisure Professional Services. (2002). 2002 Arctic Winter Games: Nuuk, Greenland and Iqaluit, Canada personal and social benefits of participation (pp. 1-58). Yellowknife: Northwest Territories.

Young, N. L., Wabano, M. J., Burke, T. A., Ritchie, S. D., Mishibinijima, D., \& Corbiere, R. G. (2013). A process for creating the Aboriginal children's health and well-being measure (ACHWM). Canadian Journal of Public Health, 104(2), e136e141. 